REGARDS

SUR LEECONOMIE ALLEMANDE

BULLETIN ECONOMIQUE DU CRAC

\section{Regards sur l'économie allemande}

Bulletin économique du CIRAC

$75 \mid 2006$

Varia

\title{
Nouveaux enjeux de la politique salariale
}

\section{René Lasserre}

\section{OpenEdition}

\section{Journals}

Édition électronique

URL : http://journals.openedition.org/rea/829

DOI : $10.4000 /$ rea. 829

ISBN : 978-2-8218-0846-1

ISSN : 1965-0787

Éditeur

CIRAC

Édition imprimée

Date de publication : 1 mars 2006

Pagination : 13-18

ISSN : 1156-8992

Référence électronique

René Lasserre, « Nouveaux enjeux de la politique salariale », Regards sur l'économie allemande [En ligne], 75 | mars 2006, document 2, mis en ligne le 19 mai 2008, consulté le 01 mai 2019. URL : http:// journals.openedition.org/rea/829; DOI : 10.4000/rea.829 


\section{Nouveaux enjeux de la politique salariale}

\begin{abstract}
René Lasserre
Après l'adoption des grandes orientations budgétaires et financières du gouvernement Merkel, les négociations salariales qui s'engagent en ce début d'année 2006 constituent un premier test important pour la nouvelle politique économique allemande. Le tour conflictuel qu'ont pris d'emblée les discussions dans les services publics où le syndicat ver.di a choisi d'engager l'épreuve de force avec les Länder sur la question de l'allongement du temps de travail des employés publics ne constitue, de ce point de vue, qu'un enjeu catégoriel circonscrit et relativement secondaire, même si son incidence sur la gestion de l'emploi public et l'assainissement budgétaire est loin d'être négligeable. Plus déterminantes sont les négociations salariales qui viennent de s'engager entre les partenaires sociaux de l'industrie des métaux car leurs résultats donneront le ton à l'ensemble des grandes branches industrielles du secteur privé. Placées sous le signe d'une nouvelle donne salariale, les négociations pourraient déboucher sur une progression des salaires et du pouvoir d'achat dont l'ampleur est encore incertaine, mais constituera une variable majeure pour l'évolution de l'emploi et de la croissance au cours des prochains mois.

Après plusieurs années de modération des salaires consentie pour sauvegarder la compétitivité externe de l'économie allemande, la reprise de l'activité semble pouvoir autoriser le desserrement de la contrainte salariale afin de soutenir la demande et réduire progressivement le chômage. Pourtant, la dépendance externe extrêmement forte de l'industrie allemande incite à la plus grande prudence sur le front des coûts salariaux, sous peine d'accélérer les réductions et les délocalisations d'emplois. De sorte que c'est moins la relance uniforme du pouvoir d'achat qu'un partage différencié des gains de productivité par une régulation salariale rénovée qui constitue l'enjeu principal de la politique salariale allemande.
\end{abstract}

\section{Vers une nouvelle donne salariale}

Après les dérapages salariaux auxquels avait donné lieu l'euphorie de l'unification au début des années 1990 et le freinage provoqué par la récession de 1992-93, la politique salariale allemande est placée depuis maintenant plus de dix ans sous le signe de la modération. Selon les données présentées par le Conseil des Sages dans son dernier rapport 2005-2006, les salaires bruts, sur la base desquels est calculé le coût salarial total, n'ont augmenté de 1994 à 2004 que de 1,5\% par an en moyenne, et de seulement 0,4\% en termes de salaire net. En termes réels, c'est-à-dire hors inflation, cette retenue salariale assumée par les syndicats dans le cadre de la politique tarifaire s'est ainsi traduite de 1995 à 2000 par une stagnation et depuis lors par une régression régulière du pouvoir d'achat. Cette évolution, conjuguée avec la montée du chômage, est considérée comme la cause principale de la stagnation persistante de la demande intérieure et du retard de croissance que l'Allemagne a affiché tout au long de la période par rapport à la moyenne européenne.

La modération salariale dont il a fait preuve pendant toute la période est de plus en plus mal vécue par le mouvement syndical qui, en dépit de cette contribution volontaire à l'impératif de compétitivité, continue de voir l'emploi diminuer sous
Dix ans

de modération salariale

Frustration et nervosité grandissantes des syndicats 
La relance salariale à l'ordre du jour?
La demande intérieure au cœur du débat de politique économique l'effet de la rationalisation et des délocalisations. En 2002, les syndicats avaient manifesté leur volonté de "rompre avec la modestie " et réussi à imposer un rattrapage salarial ponctuel, mais depuis lors la tendance est plutôt à la stratégie du compromis défensif, comme l'ont montré en 2004 l'acceptation de clauses dérogatoires dans l'industrie des métaux ou les accords sur le relèvement du temps de travail. Les tensions se sont ensuite exacerbées avec le gouvernement Schröder au printemps 2005 à propos de l'Agenda 2010 jusqu'à ce que les syndicats rentrent dans le rang à la perspective de l'échéance électorale anticipée et d'une alternance libérale. Ils ont finalement accueilli la Grande coalition avec soulagement, saluant positivement les mesures de relance de son programme de gouvernement et sa volonté marquée de faire reculer le chômage. Les syndicats n'en restent pas moins très vigilants à l'égard du point dur que constitue l'augmentation de la TVA au $1^{\mathrm{er}}$ janvier 2007, car ils redoutent qu'après plusieurs années de 'vaches maigres' elle vienne encore amputer davantage le pouvoir d'achat des salariés.

Pour les syndicats, la tentation est donc forte et, à certains égards compréhensible, de vouloir anticiper cette ponction supplémentaire sur les revenus en relevant leurs exigences salariales pour 2006. C'est ainsi qu'il faut interpréter l'objectif d'une hausse salariale de $5 \%$ dont le syndicat IG Metall s'est fait le portedrapeau à la veille de la réouverture des négociations dans l'industrie des métaux, qui compte 3400000 salariés et rassemble, avec l'automobile, l'électrotechnique et la construction mécanique, les trois principaux secteurs industriels du pays. L'objectif est ambitieux car l'accord de référence précédent signé en février 2004 à Pforzheim et qui est arrivé à expiration au $1^{\mathrm{er}}$ mars se situait très en deçà: il avait certes permis une augmentation nominale de 4,7\%, mais répartie sur 2 ans, primes ponctuelles comprises.

La stratégie d'IG Metall n'est cependant pas de simple surenchère, car elle s'inscrit dans un contexte désormais plus favorable. La nouvelle équation de la politique économique gouvernementale qui consiste à articuler et à étaler dans le temps relance de l'économie et consolidation budgétaire offre en effet une certaine marge de manœuvre politique aux syndicats. Ceux-ci peuvent légitimement plaider en faveur d'un coup de pouce salarial qui viendrait à point soutenir la demande intérieure et accélérer la reprise afin de pouvoir mieux absorber le choc redouté d'une hausse de 3 points de TVA. L'argument sera d'autant plus facilement plaidable que l'industrie de transformation des métaux affiche des performances record à l'exportation, des carnets de commandes bien remplis et une nette reprise de ses investissements. Autant de signes d'une compétitivité retrouvée, fruit d'une modération salariale et d'efforts de productivité dont les salariés pourraient opportunément percevoir les premiers dividendes.

La perspective d'un assouplissement de la politique salariale présente en outre quelque crédibilité au plan technique et revient à l'ordre du jour du débat économique, à la faveur des interrogations croissantes que suscite le bilan mitigé de la politique économique suivie depuis 10 ans. Privilégiant l'impératif de compétitivité, celle-ci s'est résolument focalisée sur l'objectif d'un partage de la valeur ajoutée plus favorable à la production et à l'investissement. Mais autant le succès est indéniable en termes de performances économiques externes, autant le prix de cette politique apparaît élevé au plan interne car il se solde par une croissance durablement ralentie, un chômage persistant et un déséquilibre préoccupant des finances publiques. De sorte que la question se trouve désormais ouvertement posée des moyens à mettre en œuvre pour régénérer une dynamique propre de croissance et enrayer cette spirale de contraction interne qui risque encore de s'accentuer avec le vieillissement de la population.

Le débat s'est ouvert au sein même du Conseil des Sages depuis la nomination récente du Professeur Peter Bofinger lequel, se démarquant de la continuité doctrinale affichée par ses collègues en faveur d'une politique de l'offre, préconise une politique plus nettement orientée sur le soutien à la demande inté- 
rieure. Dans l'avis divergent qu'il a fait figurer dans le texte du dernier rapport du Conseil, Peter Bofinger met en évidence le paradoxe flagrant qui s'établit entre le succès des réformes menées en faveur d'une amélioration des conditions de l'offre et l'ambivalence des performances macro-économiques d'ensemble qui en ont résulté. Que ce soit à travers la réforme fiscale, l'allègement tangible des prélèvements obligatoires, l'accroissement de la part des assurés dans le financement des régimes sociaux, et plus encore à la faveur de la politique de retenue salariale des partenaires sociaux, les coûts de production n'ont cessé de décroître et la rentabilité des entreprises de s'améliorer. Mais dans le même temps, le chômage s'est accru d'un million de personnes, le nombre des actifs occupés s'est réduit de 2 millions, l'accroissement annuel du potentiel de production a reculé de $2 \%$ à $1 \%$, tandis que le différentiel de croissance par rapport à la moyenne de l'UE se creusait de $1 \%$ à $1,5 \%$.

Dans ce diagnostic sans complaisance, Peter Bofinger pointe du doigt la léthargie persistante de la demande intérieure dont la croissance a été la plus faible de l'OCDE. Bien que les raisons de cette faiblesse soient multiples (taux d'épargne élevé, rétraction de l'investissement public), le recul du pouvoir d'achat réel des salaires a tenu une place déterminante : alors qu'entre 2001 et 2005 les salaires réels continuaient de croître de $0,7 \%$ en moyenne dans la zone Euro, ils diminuaient de $0,3 \%$ en Allemagne. Peter Bofinger estime qu'une politique économique trop exclusivement axée sur la compétitivité externe et la réduction des coûts salariaux présente de graves inconvénients et n'est pas tenable sur le long terme pour un grand pays comme l'Allemagne dont les deux tiers de l'activité productive restent tributaires de la demande intérieure. Elle permet certes de soutenir une activité exportatrice importante, mais sans pour autant entretenir un niveau de demande intérieure suffisant pour maintenir l'activité sur le marché domestique et contribuer au renforcement du potentiel global de l'économie. La dissociation qui s'opère entre dynamique externe et stagnation interne risque d'entretenir un processus déflationniste et d'aggraver la crise des finances publiques. Estimant qu'il est temps de sortir de cette spirale récessive, Peter Bofinger préconise de relancer la demande par une augmentation modérée des salaires qui intègre à la fois les gains de productivité et une progression de pouvoir d'achat qui respecte l'objectif de stabilité de la BCE.

C'est en partie sur ce schéma que se fonde IG Metall en affichant un objectif salarial de $5 \%$ dans lequel $2 \%$ seraient attribués au titre des gains de productivité, $2 \%$ au titre de la sauvegarde du pouvoir d'achat, le $1 \%$ supplémentaire l'étant au titre d'une participation à l'amélioration des résultats selon des modalités variables à définir. Ce coup de pouce salarial serait en outre un signal de confiance adressé en direction des ménages en faveur d'une reprise de la consommation.

\section{Un pari économiquement très risqué}

Même s'il s'appuie sur quelques bons arguments, le desserrement de la politique salariale apparaît néanmoins comme un pari économiquement risqué dans la mesure où les perspectives conjoncturelles restent incertaines quant à l'évolution des coûts et des prix. L'instabilité des prix de l'énergie doit en effet inciter à la prudence sur le front salarial car elle peut induire les "effets de second tour " contre lesquels le Président de la BCE ne cesse de mettre en garde. Mais la véritable incertitude réside dans ce que sera l'impact effectif, et pour l'instant encore largement imprévisible, de l'augmentation de la TVA à compter du $1^{\mathrm{er}}$ janvier 2007. Autant celle-ci peut justifier une politique salariale proactive qui conforte l'élan de la reprise dès le printemps 2006, autant elle ne peut laisser exclure le scénario d'un double choc récessif dans lequel la hausse des coûts de production viendrait se cumuler au renchérissement mécanique des prix à la consommation.
Desserrer la politique salariale pour sortir de l'impasse récessive

Perspectives conjoncturelles incertaines : effets de 2ème tour ou double choc? 
L'effet coût des hausses salariales l'emporte largement sur l'effet demande

L'enjeu des gains de productivité...

... et de l'emploi industriel

A

triel en Allemagne et du maintien de sa compétitivité qui se trouve posée Celui ci, de par sa structure de spécialisation très fortement orienté vers l'exportation, se trouve largement tributaire de la demande externe et du marché mondial, de sorte qu'une politique de relance salariale se heurte à des limites beaucoup plus étroites que ne le laissent entrevoir les tenants d'une politique de soutien à la demande interne. Employant encore plus du quart des actifs et réalisant près du tiers de la valeur ajoutée, il garde une force d'entraînement considérable pour l'activité interne. L'opposition entre compétitivité externe et dynamique interne est de ce point de vue artificielle dans la mesure où, dans une économie industrielle globalisée comme l'Allemagne, on peut de moins en moins dissocier le tissu productif interne de l'activité exportatrice et externalisée. Pas plus d'ailleurs qu'on ne peut séparer activité industrielle et activité de services, tant les fonctions productives s'interpénètrent et deviennent interdépendantes. A l'heure de la compétition globale, l'impératif de compétitivité reste primordial et s'impose aussi bien sur le marché externe que sur un marché interne de plus en plus largement ouvert aux opérateurs mondiaux.

\section{Poursuivre la rénovation de la régulation salariale}

Rapprocher la négociation salariale de l'entreprise...

En fait, les experts entretiennent un scepticisme persistant à l'égard de l'impact yse fine des effets induits des hausses de salaires nominales montre que, du l'importance des prélèvements, ceux-ci sont très asymétriques selon que prélèvement fiscal et des cotisations sociales, les hausses du revenu disponible quelles entraînent au profit du salarié pour augmenter sa consommation sont dence beaucoup plus lourde en termes de coût : le coût salarial global se répercute intégralement dans la masse salariale et pèse directement sur la valeur ajoutée de l'entreprise. De sorte que, dans les secteurs où la concurrence inter-
nationale est particulièrement vive, comme c'est le cas dans la plupart des branches industrielles allemandes, la pression des coûts salariaux pousse, en dépit de hausses nominales modérées, les entreprises à rationaliser leur production et à supprimer des emplois. On estime ainsi qu'en 2005, dans l'industrie
des métaux, malgré une hausse salariale contenue à 2,5\%, 50000 emplois ont été supprimés. Plus globalement, si l'on prend en compte l'évolution des qualifiprix d'une compression permanente des effectifs. Une relance prématurée des salaires dans le contexte de la reprise risque d'empêcher un renversement de tendance et de n'avoir qu'un effet limité sur l'emploi.

riale procèdent essentiellement d'un arbitrage entre salaire et emploi et se trouvent avant tout déterminees par la repartition des gains de productivité. Sur tion de négociation très rigoureuse et considère, à la différence d'IG Metall, que gains de productivité ne peuvent être intégralement répercutés sous forme pour les entreprises les plus fragiles et de fermer la porte à de nouvelles emches rendues possibles par la reprise de l'activité. S'appuyant sur plusieurs salaires qui resterait de $1 \%$ en deçà de la productivité globale devrait permettre, à la faveur de la reprise, de reconstituer une centaine de milliers d'emplois industriels et de freiner le mouvement de délocalisation.

A travers ce débat, c'est en fait la question de la sauvegarde de l'emploi industriel en Allemagne et du maintien de sa compétitivité qui se trouve posée. Celui-

Dans ce nouveau contexte, la politique salariale a perdu de sa portée en tant qu'instrument de régulation économique globale ou sectorielle, sans pour autant avoir cédé de son importance en tant que processus de partage de la ri- 
chesse produite. Elle ne peut cependant remplir cette fonction efficacement qu'en retrouvant de nouvelles marges de manœuvre, à la fois en se déplaçant vers le niveau pertinent de régulation que constitue l'entreprise et en diversifiant ses contenus. Cette exigence de flexibilité invite à poursuivre le processus de différenciation dans lequel les partenaires sociaux de la métallurgie se sont délibérément engagés en 2004 avec l'accord de Pforzheim. Celui-ci a introduit la possibilité de conclure, au niveau de l'entreprise, et dès lors que la situation économique ou la stratégie de rentabilité de cette dernière le justifient, des accords dérogatoires aux normes de la convention salariale de branche. C'est dans ce cadre qu'ont été conclus au cours de l'été 2004 les accords passés dans plusieurs usines des groupes Siemens et DaimlerChrysler engagées dans un programme de restructuration. Ces accords ont à l'époque défrayé la chronique car ils prévoyaient un relèvement de la durée conventionnelle du travail de 35 à 40 heures sans compensation salariale, et ce afin d'assurer le maintien des unités de production en Allemagne. Depuis lors, les accords dérogatoires de ce type, généralement de portée plus modeste, se sont multipliés. La branche en recense environ 380 qui instaurent généralement à titre transitoire des aménagements variés aux systèmes de rémunération : aménagement du régime des heures supplémentaires, réductions de salaires et de primes, adaptation des grilles de classification salariale, contreparties en termes de maintien d'emploi ou de recrutement d'apprentis, etc.

Il est vrai que dans le contexte de compétition industrielle et de délocalisation que connaît l'industrie manufacturière allemande, cette flexibilisation de la négociation tarifaire présente généralement une vertu défensive : les aménagements salariaux visent à l'abaissement des coûts et servent d'abord à limiter les suppressions d'emploi. Mais dès lors que la procédure qui permet la conclusion de ces «pactes d'entreprise » a été instaurée et fonctionne, rien n'empêche qu'elle puisse également servir, pour les entreprises les plus performantes ou dans un contexte économique plus favorable de reprise des affaires, à un partage plus équilibré des gains de productivité sous forme de compléments de rémunération liés aux résultats de l'entreprise. La décentralisation de la négociation favorise des régulations de proximité qui, au delà des normes de productivité moyenne de la branche, permettent de développer des politiques de rémunération dynamiques, à la fois motivantes pour les salariés et génératrices de revenus supplémentaires dans l'économie domestique. Face à des contraintes fortes de compétitivité externe et des charges indirectes élevées au plan interne, la décentralisation de la politique salariale à l'intérieur des branches présente d'incontestables avantages. A l'intérieur d'un cadre conventionnel qui garantit la cohérence du marché de la main d'oeuvre, des qualifications et des conditions de travail de la branche, elle assure un partage plus fin et une meilleure allocation de la valeur ajoutée que ne peut le faire une régulation sectorielle globale et uniforme.

Dans les négociations qui viennent de s'ouvrir, la politique salariale de la métallurgie pourrait évoluer dans cette voie à la faveur de la proposition d'IG Metall d'introduire dans l'accord des clauses relatives à l'attribution de primes annuelles modulables liées aux résultats des entreprises. II s'agit certes pour IG Metall d'obtenir par ce biais un bonus salarial qui permette d'aller au delà de l'arbitrage habituel sur les gains de productivité pour lequel le patronat campe sur des positions techniques assez fermes. Si toutefois Gesamtmetall décidait de mettre à profit cette ouverture, la négociation pourrait déboucher sur la mise en place, dans la branche-phare de l'industrie allemande, d'un dispositif conventionnel inédit. Sur ce point, la négociation aura valeur de test car elle pourrait marquer une avancée significative de la rémunération liée aux résultats qui, jusqu'à présent est encore peu développée en Allemagne. Un second projet inscrit au programme de la négociation consiste à promouvoir par voie d'accords d'entreprises des programmes de qualification-innovation dont les
... pour générer de nouvelles dynamiques salariales

Explorer de nouveaux champs et lever la rigidité des salaires d'embauche 
modalités restent pour l'instant au stade exploratoire, mais visent aussi à mettre à profit les marges de progrès que peut offrir l'entreprise.

Autant de nouveaux chantiers que la régulation d'entreprise permet de mettre en oeuvre pour faire prévaloir sur le terrain des solutions pragmatiques fondées sur une logique de réciprocité. Ainsi devraient pouvoir être progressivement levés, par la voie de l'expérimentation, certains blocages persistants de la politique salariale comme celui de la rigidité des salaires minima. II serait alors possible de faire la preuve que la flexibilité des salaires d'embauche constitue une alternative réelle à l'arsenal complexe de mesures en faveur du travail aidé, lesquelles s'avèrent peu efficaces pour résorber le chômage, génèrent des trappes à inactivité, et se révèlent ainsi très coûteuses en termes de charges indirectes pesant sur la rémunération du travail normal.

AVEC LES NEGOCIATIONS QUI S'OUVRENT DANS LA METALLURGIE, la politique salariale aborde une nouvelle étape sur la voie de sa modernisation. Après les expériences ponctuelles de négociations dérogatoires amorcées dans les années 90 , l'accord de Pforzheim a constitué une première avancée d'envergure vers la décentralisation de la négociation contractuelle. A l'origine, c'est en grande partie pour couper court aux tentations du législateur d'introduire dans la loi sur les conventions collectives (Tarifvertragsgesetz) des dispositions sur la négociation dérogatoire d'entreprise que les partenaires sociaux de la métallurgie se sont engagés résolument dans cette démarche. Mais jusqu'à présent, les nouvelles formes de régulation qu'ils ont mises en œuvre ont davantage servi à sauvegarder l'emploi et l'outil de production qu'elles n'ont fait la preuve de leur capacité à infléchir la tendance à la régression des acquis et à générer du progrès social. Dans un contexte de retour à la croissance, la tentation pourrait être grande d'en revenir aux règles du jeu classiques du partage social. L'analyse des enjeux de la politique salariale montre cependant que les contraintes économiques restent fortes, que les marges de manœuvre sont étroites et que les voies d'un renouveau dans le partage salarial exigent la recherche de régulations innovantes et différenciées impliquant les acteurs au plus près du terrain où se crée la richesse.

\section{Indications bibliographiques:}

BISPINK R., "Tarifsstandards unter Druck. Tarifpolitischer Jahresbericht 2004 », in WSI Mitteilungen 2/2005

BISPINK R., « Tarifpolitischer Halbjahresbericht 2004. Zwischenbilanz der Lohn- und Gehaltsrunde 2005 ", in WSI Mitteilungen 7/2005

BISPINK R., « Tarifpolitischer Jahresbericht 2005 : Gemischte Bilanz. Reallohnverluste überwiegen », in WSI Mitteilungen 2/2006

LATTARD A., "Négociation collective : quel avenir pour la convention de branche ? » in BOURGEOIS I. (dir), Le modèle social allemand en mutation, Cergy, CIRAC, 2005 LESCH H., « Lohnpolitik und Beschäftigung in Deutschland », in IW-trends 4/2005 SACHVERSTÄNDIGENRAT, Die Chance nutzen - Reformen mutig voranbringen. Jahresgutachten 2005/06.

SACHVERSTÄNDIGENRAT, Erfolge im Ausland, Herausforderungen in Inland. Jahresgutachten 2004/05. 